\title{
All-Solution-Processed, Oxidation-Resistant Copper Nanowire Networks for Optoelectronic Applications with Year-Long Stability
}

Sevim Polat Genlik, Dogancan Tigan, Yusuf Kocak, Kerem Emre Ercan, Melih Ogeday Cicek, Sensu Tunca, Serkan Koylan, Sahin Coskun, Emrah Ozensoy, and Husnu Emrah Unalan*

Cite This: ACS Appl. Mater. Interfaces 2020, 12, 45136-45144

Read Online

\section{ACCESS | Llll Metrics \& More | 国 Article Recommendations | (I) Supporting Information}

ABSTRACT: Copper nanowires ( $\mathrm{Cu}$ NWs) hold promise as they possess equivalent intrinsic electrical conductivity and optical transparency to silver nanowires (Ag NWs) and cost substantially less. However, poor resistance to oxidation is the historical challenge that has prevented the large-scale industrial utilization of $\mathrm{Cu}$ NWs. Here, we use benzotriazole (BTA), an organic corrosion inhibitor, to passivate $\mathrm{Cu}$ NW networks. The stability of BTApassivated networks under various environmental conditions was monitored and compared to that of bare $\mathrm{Cu}$ NW control samples. BTA passivation greatly enhanced the stability of networks without deteriorating their optoelectronic performance. Moreover, to demonstrate their potential, BTA-passivated networks were successfully utilized in the fabrication of a flexible capacitive tactile

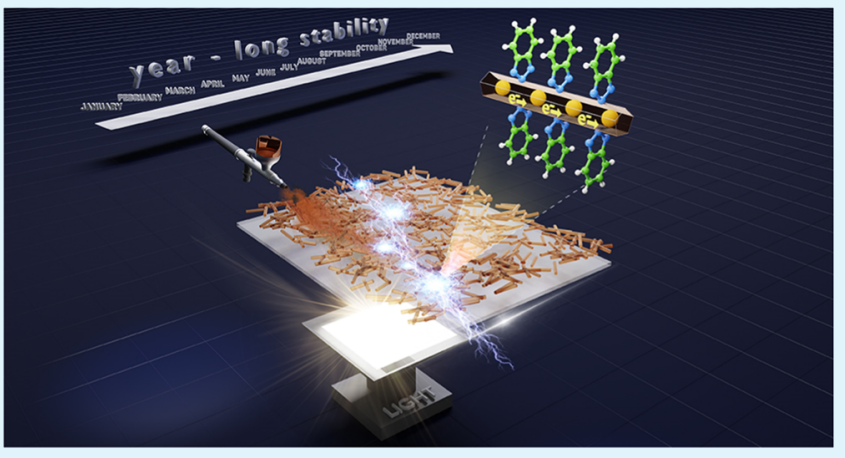
sensor. This passivation strategy has a strong potential to pave the way for large-scale utilization of Cu NW networks in optoelectronic devices.

KEYWORDS: copper nanowires, benzotriazole, oxidation stability, passivation, transparent conducting electrodes, flexible tactile sensors

\section{INTRODUCTION}

Transparent electrodes are key components of many optoelectronic devices such as touch panels, photovoltaic devices, light-emitting diodes, and transparent thin-film heaters. ${ }^{1,2}$ Although the majority of commercial products in the market are based on indium tin oxide (ITO) thin films, high processing costs, brittleness, and indium scarcity renders ITO-based systems undesirable in the next generation of optoelectronic devices requiring mechanical and functional flexibility. Therefore, much effort has been devoted to the development of mechanically flexible, large-scale processable, low-cost, and lightweight alternative materials for transparent and conducting electrodes. Ag NW networks appear to be the most promising alternative due to their equivalent or better optoelectronic performance than that of ITO. ${ }^{3-6} \mathrm{Cu}$ NWs also hold great potential because they possess comparable intrinsic electrical conductivity to $\mathrm{Ag} \mathrm{NWs}\left(\rho_{\mathrm{Ag}}=1.6 \times 10^{-8} \Omega \mathrm{m}, \rho_{\mathrm{Cu}}\right.$ $\left.=1.7 \times 10^{-8} \Omega \mathrm{m}\right)$ and are lower in cost. ${ }^{7}$ Successful integration of $\mathrm{Cu}$ NW networks as transparent conducting electrodes into a variety of functional prototype devices, such as organic photovoltaics, ${ }^{8-10}$ organic light-emitting diodes, $^{11-13}$ touch sensors, ${ }^{14,15}$ and transparent thin-film heaters, ${ }^{16-18}$ has already been demonstrated. However, poor oxidation stability of $\mathrm{Cu}$ NWs presents a major barrier in front of their utilization in mass-produced commercial devices. Therefore, the development of an efficient method to protect
$\mathrm{Cu}$ NW networks from atmospheric corrosion without deteriorating their optoelectronic performance is a crucial challenge of paramount significance. Various routes have been proposed to solve the stability issue. These include coating $\mathrm{Cu}$ NWs with metals, ${ }^{7,8,18-20}$ metal oxides, ${ }^{9,16,21,22}$ carbon, nitridebased materials ${ }^{23-25}$ and polymers. ${ }^{10,26}$ Despite these studies, development of $\mathrm{Cu}$ NW-based network fabrication routes that can (i) enable highly efficient long-term corrosion protection, (ii) preserve the optoelectronic performance of the network (particularly sheet resistance, transmittance, and haze), and (iii) allow quick, affordable, and simple means of mass production of large-area electrodes remain elusive.

It is well known that bulk $\mathrm{Cu}$ is prone to oxidation. Thus, numerous methods have been proposed to increase its oxidation stability. For instance, studies have reported the use of organic corrosion inhibitors (e.g., azoles, amines, amino acids, self-assembled monolayers) that are effective in enhancing oxidation resistance of bulk copper. ${ }^{27,28}$ Among these, azoles such as benzotriazole (BTA), benzimidazole,

Received: June 28, 2020

Accepted: September 8, 2020

Published: September 8, 2020 

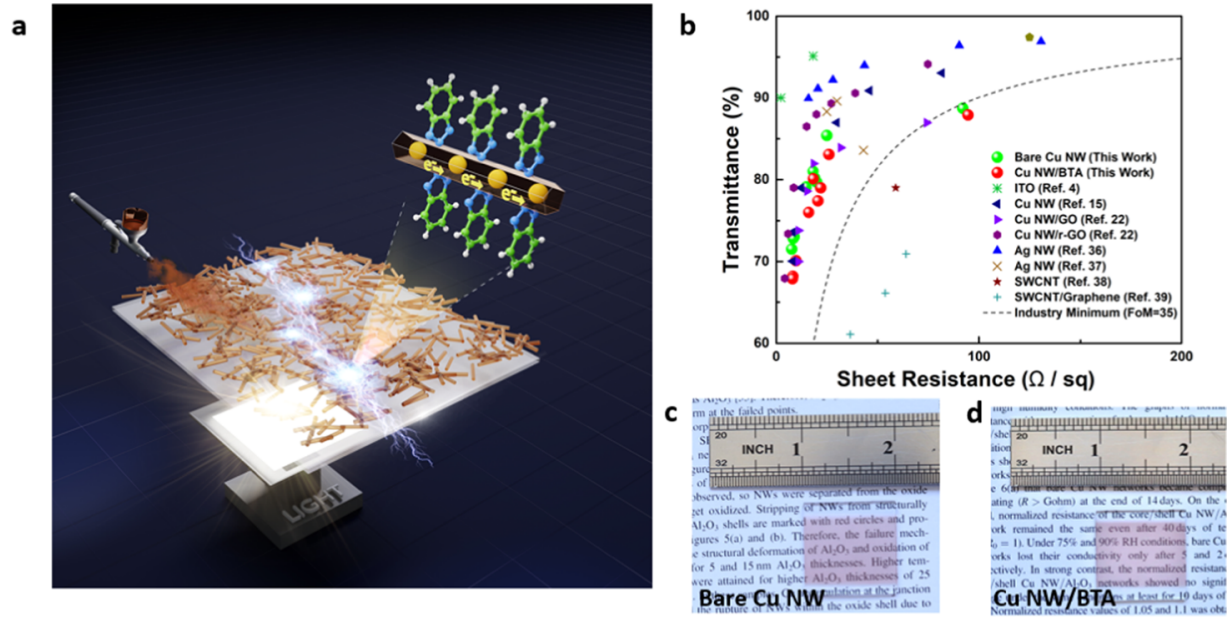

Figure 1. (a) Schematic illustration for the fabrication of transparent and conducting Cu NW networks. (b) Sheet resistance versus transmittance (at $550 \mathrm{~nm}$ ) of $\mathrm{Cu} \mathrm{NW}$ networks (before and after BTA passivation) in comparison to various transparent electrodes reported in the literature including ITO. Photographs of (c) bare and (d) BTA-passivated Cu NW networks.

tetrazole, pyridazole, other nitrogen-containing heterocyclic compounds, and their derivatives have been demonstrated as highly efficient corrosion inhibitors for bulk $\mathrm{Cu}$ and its alloys. In particular, outstanding corrosion inhibition efficiency for bulk $\mathrm{Cu}$ was reported using BTA. The stabilization of $\mathrm{Cu}$ using BTA has been ascribed to the formation of a polymeric barrier film that is insoluble in water and many organic solvents. ${ }^{29-35}$ BTA was also used for improving the stability of $\mathrm{Cu}$ nanoparticles $^{33}$ and $\mathrm{Cu}$ NWs. ${ }^{34}$ Wiley's group demonstrated the first use of BTA for improving the stability of $\mathrm{Cu} N W$ networks and their consequent use as transparent electrodes in organic photovoltaic devices. ${ }^{34}$ However, a $20 \%$ increase in the sheet resistance of $\mathrm{Cu}$ NW networks was reported upon BTA deposition, probably due to the use of the aqueous soaking solution for BTA deposition. ${ }^{34}$

In the current study, BTA was utilized to enhance the longterm oxidation stability of $\mathrm{Cu} \mathrm{NW}$ networks without degrading their performance. High-aspect-ratio $\mathrm{Cu}$ NWs synthesized through a hydrothermal method were used to deposit optically transparent and electrically conducting $\mathrm{Cu}$ NW networks. Optoelectronic properties of $\mathrm{Cu} \mathrm{NW}$ networks under ambient conditions as well as under operationally harsh environments were systemically investigated and compared to that of bare $\mathrm{Cu}$ NW control samples. A clear understanding of the limits of BTA passivation on $\mathrm{Cu}$ NW networks through detailed spectroscopic and structural analysis was provided. At last, BTA-passivated networks were successfully utilized in transparent and flexible capacitive tactile sensors that can be integrated into a variety of devices.

\section{RESULTS AND DISCUSSION}

Following the synthesis of $\mathrm{Cu}$ NWs through a hydrothermal method, $\mathrm{Cu} \mathrm{NW}$ networks were fabricated via a simple spray deposition method. Figure 1a schematically illustrates the fabrication process of transparent and conducting $\mathrm{Cu}$ NW networks. Note that this simple yet effective production method can readily be utilized to manufacture large-scale electrodes with high speed, high quality, and low production cost. Furthermore, this deposition method is substrate-neutral as it can be used to coat any substrate. Following fabrication, $\mathrm{Cu}$ NW networks were passivated with BTA that was practiced through spin coating of a 3 wt \% BTA-ethanol solution, followed by an ethanol rinse and drying.

To investigate the stability of $\mathrm{Cu} \mathrm{NW}$-based electrodes, it is crucial to elucidate the structural and functional characteristics of individual NWs as well as the entire NW networks. The average diameter and length of synthesized $\mathrm{Cu}$ NWs were 45.4 $\pm 17.4 \mathrm{~nm}$ and $49.3 \pm 19.9 \mu \mathrm{m}$, respectively (Figure S1). Prior to annealing, all bare $\mathrm{Cu}$ NW networks were found to be nearly insulating with sheet resistance values on the order of $10^{6} \Omega / \mathrm{sq}$ with optical transmittance values higher than $70 \%$ (at $550 \mathrm{~nm}$ ). Annealing was performed to remove the residual surfactant (PVP) layer and the residual capping agent (HDA) and to improve the electrical contacts by fusing nanowire-nanowire junctions. After annealing, the typical sheet resistance of bare $\mathrm{Cu}$ NW networks was found to be $30 \pm 9 \Omega$ /sq at an optical transmittance (at $550 \mathrm{~nm}$ ) of $86 \%$. The sheet resistance and transmittance values of networks can be simply controlled with NW density. The change in sheet resistance with the optical transmittance of $\mathrm{Cu} \mathrm{NW}$ networks both before and after BTA passivation in comparison to several other studies in the literature on transparent electrodes is provided in Figure $1 \mathrm{~b}$. BTA passivation resulted in a minute increase in sheet resistance (of $1 \Omega / \mathrm{sq}$ ) and a slight decrease in optical transmittance (of $2 \%$ ). Hence, no significant changes were detected due to the passivation process and BTA-passivated networks showed comparable figure-of-merit transparency and sheet resistance to those of bare $\mathrm{Cu}$ NW networks (Figure $1 \mathrm{~b}$ ).

X-ray photoelectron spectroscopy (XPS) analysis was performed to determine the initial compositions of the asprepared and BTA-passivated networks. It was found that the as-deposited bare $\mathrm{Cu} \mathrm{NW}$ networks were mostly in the oxidic form (i.e., predominantly $\mathrm{CuO}$ with a minor contribution from $\mathrm{Cu}_{2} \mathrm{O}$ ) evident by the appearance of a high $\mathrm{Cu} 2 \mathrm{p}_{3 / 2}$ binding energy (B.E.) shoulder at $934.0 \mathrm{eV}$ as well as the strong satellite peak at ca. $944 \mathrm{eV}$ (Figure S2a). Obviously, without BTA protection, metallic $\mathrm{Cu}$ is not likely to survive on the surface even under ambient conditions during the short time period between network fabrication and XPS measurements. However, the high electrical conductivity of bare $\mathrm{Cu}$ NW networks signifies that the $\mathrm{Cu}$ NWs are not entirely oxidized and metallic $\mathrm{Cu}$ possibly resides below the $\mathrm{CuO}$ and $\mathrm{Cu}_{2} \mathrm{O}$ layers on the surface. Moreover, it should be noted that the 
presence of $\mathrm{Cu}_{2} \mathrm{O}$ on the outermost surface cannot be ruled out, as its $\mathrm{Cu} 2 \mathrm{p}$ signals overlap with those of $\mathrm{Cu}$. Overall, the structure of fresh, bare $\mathrm{Cu}$ NW networks was possibly in the form of either a $\mathrm{CuO}$ shell with a $\mathrm{Cu}$ core and/or a $\mathrm{CuO}+$ $\mathrm{Cu}_{2} \mathrm{O}$ shell with a $\mathrm{Cu}$ core. In sharp contrast, it was found that there is no $\mathrm{CuO}$ on BTA-passivated $\mathrm{Cu} \mathrm{NW}$ networks due to the absence of high B.E. $\mathrm{Cu} 2 \mathrm{p}_{3 / 2}$ shoulder at $934.0 \mathrm{eV}$, and the strong satellite at $944 \mathrm{eV}$ (Figure S2b). As a result, the structure of BTA-passivated $\mathrm{Cu} \mathrm{NW}$ networks is possibly in the form of prominently metallic $\mathrm{Cu}$ with some adsorbed $\mathrm{O}$ (due to $\mathrm{H}_{2} \mathrm{O}$ or $-\mathrm{OH}$ ) and perhaps very small amounts of $\mathrm{Cu}_{2} \mathrm{O}$.

At last, it should be noted that the stability of transparent and conducting metallic NW networks strongly depends on the amount of NWs forming the network. Although there exists a vast number of studies in the literature on the stability of metallic NW networks, most of these studies fail to report the NW density of the fabricated networks. This makes relative stability comparisons of the different types of metal NW networks difficult. In the current study, the density of $\mathrm{Cu} \mathrm{NW}$ networks was calculated for each network as areal mass density (amd) in units of $\mathrm{mg} / \mathrm{m}^{2}$. The amd calculation details are provided in Supporting Information Figure S3a,b. Networks with amd values of $120-162 \mathrm{mg} / \mathrm{m}^{2}$ were used for all stability tests reported here.

Oxidation Resistance of Networks under Ambient Conditions. To compare the oxidation resistance performance under ambient conditions, sheet resistances of bare $\mathrm{Cu}$ NW and BTA-passivated Cu NW networks were monitored for an extended period of time (i.e., 1 year). Networks were prepared with similar NW densities of $124-148 \mathrm{mg} / \mathrm{m}^{2}$ to eliminate the effect of the NW density on the degradation of electrodes. During measurements, all electrodes were kept under atmospherically regulated conditions (i.e., in air with an average temperature of $22 \pm 2{ }^{\circ} \mathrm{C}$ and average humidity of 25 $\pm 5 \% \mathrm{RH})$. The normalized sheet resistance-the ratio of the sheet resistance to the initial sheet resistance $\left(R / R_{0}\right)$-was used as a test standard. Figure $2 \mathrm{a}$ shows the graph of

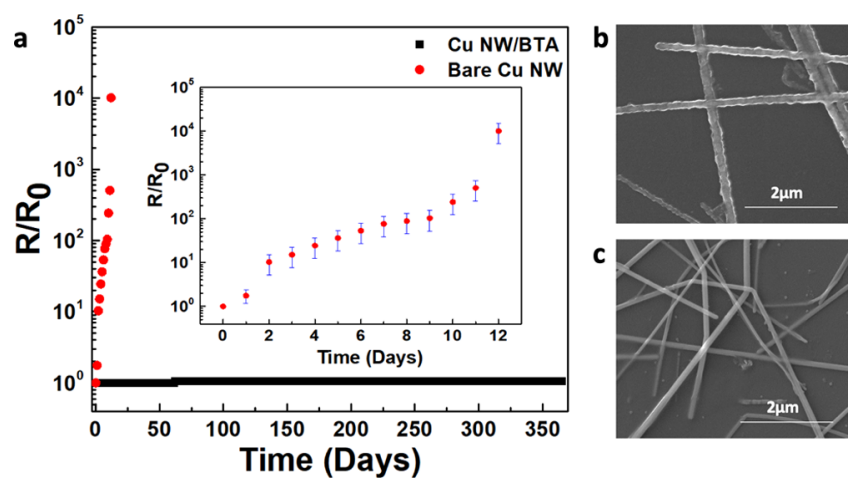

Figure 2. (a) Change in the sheet resistance of bare and BTApassivated $\mathrm{Cu}$ NW networks under ambient conditions. SEM images of (b) bare $\mathrm{Cu} \mathrm{NW}$ networks and (c) BTA-passivated $\mathrm{Cu}$ NW networks after one-year exposure to ambient conditions.

normalized sheet resistance versus time for bare and BTApassivated $\mathrm{Cu}$ NW networks. Bare $\mathrm{Cu}$ NWs lost their conductivity within 12 days, while the sheet resistance of BTA-passivated networks increased by a factor of only 1.1 at the end of one year. Scanning electron microscopy (SEM) images of bare and BTA-passivated $\mathrm{Cu}$ NW networks after a one-year test period are shown in Figure $2 b, c$, respectively. The surface roughness of bare $\mathrm{Cu}$ NWs was found to increase dramatically. In sharp contrast, BTA-passivated $\mathrm{Cu} \mathrm{NWs}$ preserved their smoothness and no prominent morphological changes were observed. XPS analysis of bare $\mathrm{Cu}$ NW networks upon exposure to ambient conditions during a year-long exposure to ambient conditions suggested that the loss in electrical conductivity and the change in morphology of $\mathrm{Cu}$ NWs were due to complete oxidation of $\mathrm{Cu}$ to $\mathrm{CuO}$. This was evidenced by the appearance of characteristic strong satellite peaks at 943.6 and $962.0 \mathrm{eV}$ (Figure S4).

Oxidation Resistance of Networks at High Relative Humidity Conditions. To accelerate their degradation, $\mathrm{Cu}$ NW electrodes were also stored under high relative humidity (RH) environments (e.g., 75 and 90\% RH) for 10 days. Sheet resistance values along with the structural properties of the electrodes were monitored via conductivity measurements and XPS and transmission electron microscopy (TEM) analyses. Networks prepared for these studies had similar NW densities of $148-162 \mathrm{mg} / \mathrm{m}^{2}$. Increased humidity severely affected the stability of bare $\mathrm{Cu} \mathrm{NW}$ networks. Although bare $\mathrm{Cu} \mathrm{NW}$ networks lost their electrical conductivity due to oxidation in 12 days under ambient conditions, it took approximately 5 days for the complete loss of conductivity under $75 \% \mathrm{RH}$ (Figure 3a). The corrosion rate was much higher under $90 \%$ $\mathrm{RH}$ for bare $\mathrm{Cu}$ NW networks so that they became nonconducting only after 2 days (Figure 3a). In sharp contrast, the resistance change for BTA-passivated $\mathrm{Cu}$ NW networks was negligible at the end of 10 days. The chemical composition of NWs after exposure to high humidity conditions was determined through XPS analysis. Characteristic strong satellite peaks appearing at 942.5 and $962.2 \mathrm{eV}$, as well as $\mathrm{Cu} 2 \mathrm{p}_{3 / 2}$ and $\mathrm{Cu} 2 \mathrm{p}_{1 / 2}$ peaks at 933.3 and $953.1 \mathrm{eV}$, respectively, in the XPS spectrum revealed that the bare $\mathrm{Cu}$ NWs were oxidized predominantly to $\mathrm{CuO}$ with a minor contribution from $\mathrm{Cu}_{2} \mathrm{O}^{32}$ (Figure S5). SEM and TEM images provided in Figure 3b,c, respectively, reveal nanoparticle formation on the surface of the bare $\mathrm{Cu}$ NWs upon exposure to high humidity conditions. Since the polycrystalline oxide layer forms throughout the surface of NWs, the formed grain boundaries and other types of defects act as nucleation sites for the formation of $\mathrm{CuO} / \mathrm{Cu}_{2} \mathrm{O}$ nanoparticles. ${ }^{36}$

Oxidation Resistance of Networks at Elevated Temperatures. Since typical optoelectronic device fabrication processes involve multiple annealing steps, $\mathrm{Cu} \mathrm{NW}$ networks should be thermally stable at these elevated temperatures. To compare the thermal stability of bare and BTA-passivated $\mathrm{Cu}$ NW networks, samples were calcined at 75,125 , and $150{ }^{\circ} \mathrm{C}$ in air. The average initial sheet resistance of these $\mathrm{Cu} N W$ networks was $19 \pm 3.8 \Omega / \mathrm{sq}$, while the initial areal mass density of networks was in the range of $120-150 \mathrm{mg} / \mathrm{m}^{2}$. For all thermal aging tests, samples were directly exposed to the chosen temperatures in air without a heating ramp. Figure $3 \mathrm{~d}-$ $f$ shows the change in the sheet resistance for both bare and BTA-passivated $\mathrm{Cu}$ NW networks as a function of the time at calcination temperatures of 75,125 , and $150{ }^{\circ} \mathrm{C}$, respectively. At $75{ }^{\circ} \mathrm{C}$, BTA-passivated networks showed superior stability with respect to the bare networks (Figure 3d). Although it took approximately $250 \mathrm{~min}$ for the bare $\mathrm{Cu} \mathrm{NW}$ network to be totally nonconductive at $75{ }^{\circ} \mathrm{C}$, similar failure occurred within $50 \mathrm{~min}$ at $125^{\circ} \mathrm{C}$ and only within $20 \mathrm{~min}$ at $150{ }^{\circ} \mathrm{C}$. On the other hand, BTA-passivated $\mathrm{Cu}$ NW networks showed better resistance against oxidation compared to the bare $\mathrm{Cu}$ 

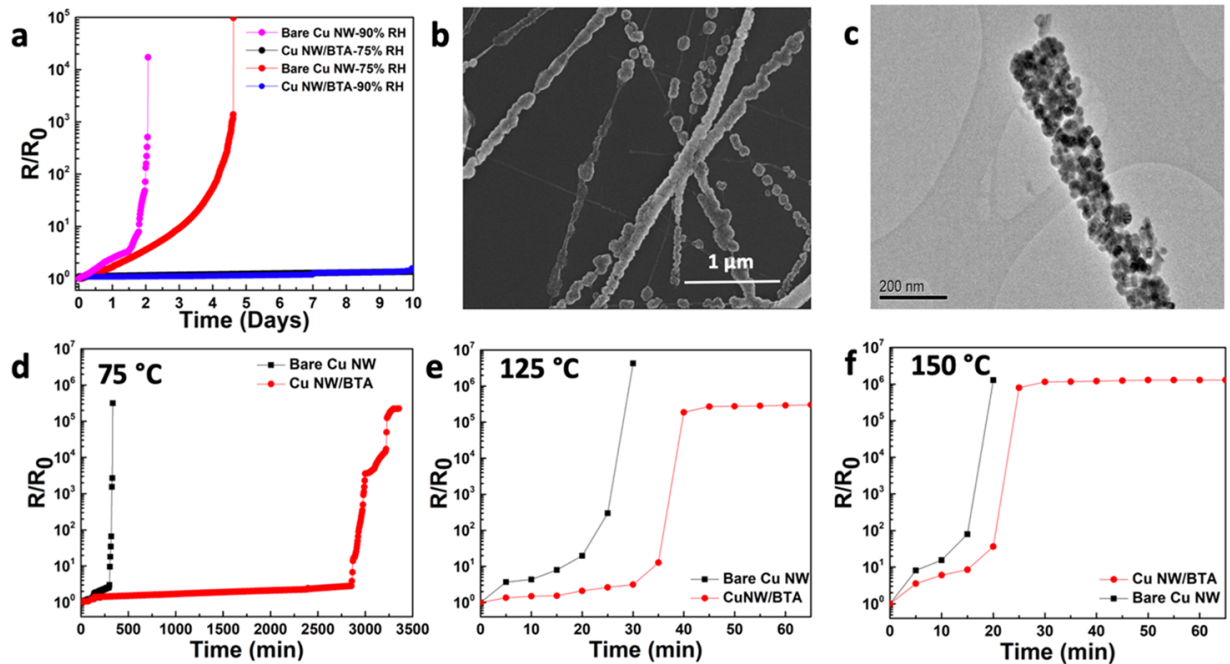

Figure 3. (a) Change in the sheet resistance of bare and BTA-passivated Cu NW networks kept at 75 and $90 \%$ RH for 10 days. (b) SEM and (c) TEM images of bare $\mathrm{Cu}$ NW networks aged under $75 \pm 5 \% \mathrm{RH}$. Change in the sheet resistance of bare Cu NW and BTA-passivated Cu NW networks as a function of the calcination time in air at (d) 75 , (e) 125 , and (f) $150{ }^{\circ} \mathrm{C}$ (lines are for visual aid).
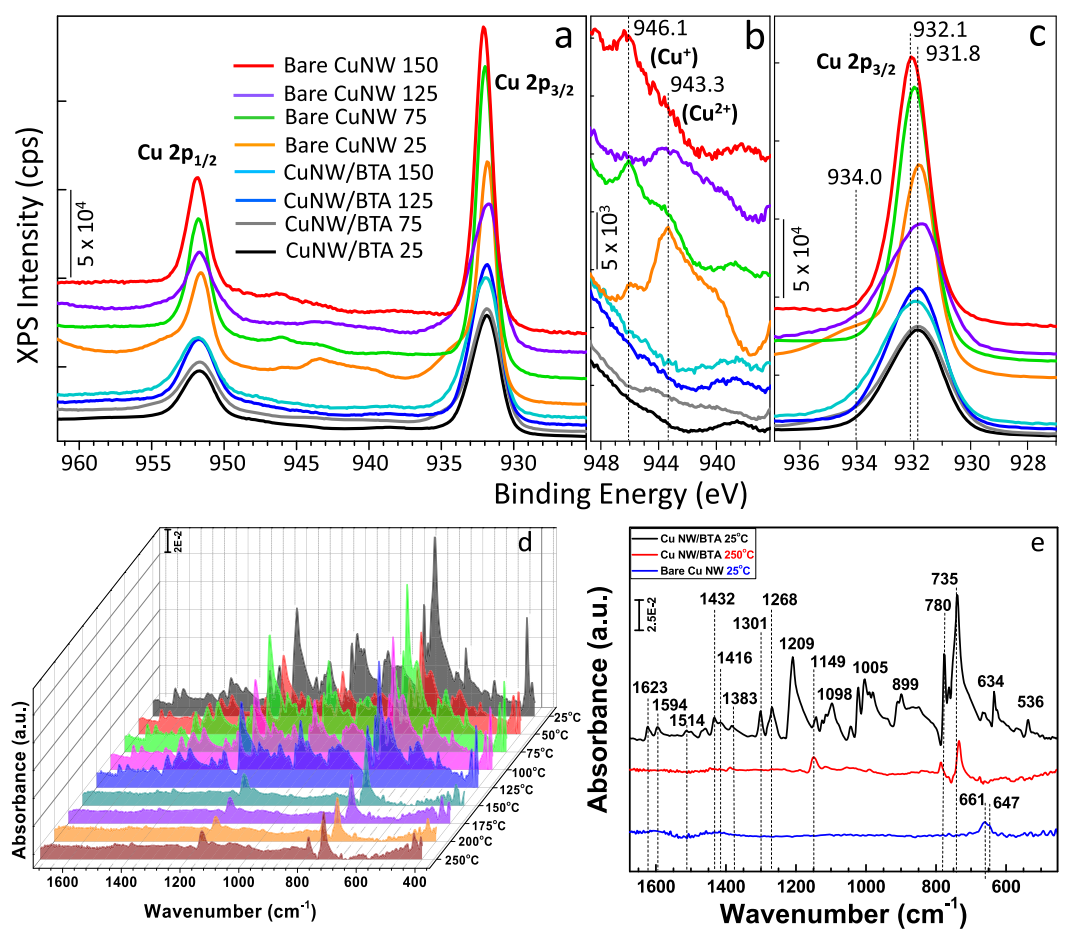

Figure 4. (a-c) Cu 2p XPS spectra of bare and BTA-passivated Cu NW networks at $25^{\circ} \mathrm{C}$ and compared with networks calcined at 75,125 , and $150^{\circ} \mathrm{C}$. (d) Attenuated total reflection-infrared (ATR-IR) data obtained after 5 min calcination of BTA-passivated Cu NW networks in air within $25-150{ }^{\circ} \mathrm{C}$. (e) ATR-IR spectra obtained for BTA-passivated Cu NWs at $25{ }^{\circ} \mathrm{C}$ (black spectrum), after degradation of the BTA coating at $250{ }^{\circ} \mathrm{C}$ (red spectrum) and bare $\mathrm{Cu}$ NWs (blue spectrum) at $25{ }^{\circ} \mathrm{C}$.

NW samples (Figure 3e,f). Typical annealing temperatures involved in the fabrication of optoelectronic devices lie around $100{ }^{\circ} \mathrm{C}$, and hence, BTA-passivated $\mathrm{Cu} \mathrm{NW}$ networks will survive these processes. Moreover, the rate of change in resistance values decreased significantly before networks became completely insulating for all of the calcination temperatures investigated (Figure $3 \mathrm{~d}-\mathrm{f}$ ). This very slow rate could be attributed to the gradual loss of BTA following the rapid loss at the initial stage. ${ }^{37}$

To determine the oxidation states of both bare and BTApassivated $\mathrm{Cu}$ NW networks, XPS analysis was performed following $5 \mathrm{~min}$ calcination at 75,125 , and $150{ }^{\circ} \mathrm{C}$. Three major factors may tend to change the oxidation state of $\mathrm{Cu}$ in the presence of atmospheric oxygen and thermal activation provided by calcination/heat. Firstly, atmospheric $\mathrm{O}_{2}$ can be activated and dissociated on the $\mathrm{Cu}$ surface, leading to the oxidation of $\mathrm{Cu}$. This was observed for the freshly prepared bare $\mathrm{Cu} \mathrm{NW}$ networks at $25{ }^{\circ} \mathrm{C}$ (Figure 4b), where the surface of the $\mathrm{Cu}$ NWs was determined to be oxidized to predominantly $\mathrm{Cu}^{2+}$ (i.e., $\mathrm{CuO}$ ) species with a smaller contribution from $\mathrm{Cu}^{+}$(i.e., $\mathrm{Cu}_{2} \mathrm{O}$ ) species. Oxidation of metallic $\mathrm{Cu}$ to $\mathrm{Cu}^{2+}$ and $\mathrm{Cu}^{+}$is clearly discernible by the 
presence of the satellite features in Figure $4 b$, as well as the high B.E. shoulder at $934.0 \mathrm{eV}$ in the $\mathrm{Cu} 2 \mathrm{p}_{3 / 2}$ signal (Figure 4c). ${ }^{32}$ The second factor influencing the $\mathrm{Cu}$ oxidation state during the thermal treatment can be the desorption/loss of lattice oxygen from $\mathrm{CuO}$ and $\mathrm{Cu}_{2} \mathrm{O}$ structures. In contrast to the first factor, this second factor tends to reduce $\mathrm{Cu}^{2+}$ to $\mathrm{Cu}^{+}$ and-less likely- $\mathrm{Cu}^{+}$to $\mathrm{Cu}$ species. Finally, as the third factor, oxidation of the surface $\mathrm{C}$ and $\mathrm{N}$ residues originating from hexadecylamine (HDA) and poly(vinylpyrrolidone) (PVP) used in the synthesis and incorporation of $\mathrm{CuO}$ and $\mathrm{Cu}_{2} \mathrm{O}$ lattice oxygen to the surface organic functionalities may also tend to reduce $\mathrm{CuO}$ to $\mathrm{Cu}_{2} \mathrm{O}$ and less likely $\mathrm{Cu}_{2} \mathrm{O}$ to $\mathrm{Cu}$. Hence, there are multiple opposing processes favoring oxidation or reduction of the $\mathrm{Cu}$ NW surface upon calcination in air. This is also apparent in the oscillatory behavior of the oxidation state of the bare $\mathrm{Cu}$ NWs upon calcination in air, where at $25{ }^{\circ} \mathrm{C}$, the bare $\mathrm{Cu} N W$ surface is dominated by $\mathrm{CuO}$ with a minor contribution from $\mathrm{Cu}_{2} \mathrm{O}$ (note the minor 945.1 $\mathrm{eV} \mathrm{Cu}_{2} \mathrm{O}$ satellite feature at $25^{\circ} \mathrm{C}$ ). However, upon calcination at $75{ }^{\circ} \mathrm{C}$, a partial reduction was observed and bare $\mathrm{Cu} \mathrm{NWs}$ tend to reveal mostly $\mathrm{Cu}_{2} \mathrm{O}$ species, evident by the loss of the high B.E. $\mathrm{Cu} 2 \mathrm{p}_{3 / 2}$ shoulder at $934.0 \mathrm{eV}$, sharpening of the $\mathrm{Cu}$ $2 \mathrm{p}_{3 / 2}$ signal, a decrease in the $943.3 \mathrm{eV} \mathrm{CuO}$ satellite feature, and strengthening of the $946.1 \mathrm{eV} \mathrm{Cu}_{2} \mathrm{O}$ satellite feature (Figure 4b). Upon calcination at a higher temperature of 125 ${ }^{\circ} \mathrm{C}, \mathrm{Cu}_{2} \mathrm{O}$ was reoxidized to $\mathrm{CuO}$, which was readily visible

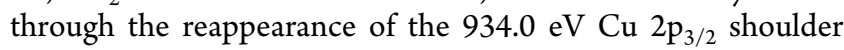
and $943.3 \mathrm{eV}$ satellite feature. Further calcination at $150{ }^{\circ} \mathrm{C}$ tended to partially reduce the $\mathrm{Cu}$ NWs back to mostly $\mathrm{Cu}_{2} \mathrm{O}$ (see the satellite in Figure 4b). These oxidation state variations of bare $\mathrm{Cu} \mathrm{NW}$ network as a function of temperature was also in accordance with the surface atomic oxygen content determined via XPS measurements (see Figure S6a).

When $\mathrm{Cu}$ NWs were passivated with BTA, Cu sites tend to protect their metallic nature in air for all investigated temperatures $\left(25-150{ }^{\circ} \mathrm{C}\right.$ ) (Figure $4 \mathrm{a}-\mathrm{c}$ ). The lack of a significant amount of $\mathrm{CuO}$ was evident due to the $\mathrm{Cu} 2 \mathrm{p}_{3 / 2}$ B.E. located at $931.9 \mathrm{eV}$ and the absence of a high B.E. shoulder at $934.0 \mathrm{eV} .^{32}$ The presence of a significant amount of $\mathrm{Cu}_{2} \mathrm{O}$ could also be readily ruled out by considering the lack of a satellite peak at $944 \mathrm{eV}$ and more importantly by considering the minuscule concentration of surface atomic oxygen (ca. $2 \%$ ) determined by XPS (Figure S6b). It is obvious that BTA provided an excellent oxidation resistance for metallic $\mathrm{Cu}$ sites within $25-150{ }^{\circ} \mathrm{C}$ in air for moderate time periods.

To demonstrate the thermal stability of BTA on $\mathrm{Cu}$ NW networks, passivated networks were monitored through ATRIR spectroscopy upon 5 min calcination within a temperature range of $25-250{ }^{\circ} \mathrm{C}$. Figure $4 \mathrm{~d}$ shows that the BTA overlayer remains intact after calcination in air up to $125{ }^{\circ} \mathrm{C}$, while it drastically decomposes after calcination at $150{ }^{\circ} \mathrm{C}$.

Detailed ATR data for the BTA-passivated $\mathrm{Cu}$ NW networks calcined at $25{ }^{\circ} \mathrm{C}$ (black spectrum in Figure 4e) are in very good agreement with the former studies. ${ }^{32}$ It was apparent that after calcination at $150{ }^{\circ} \mathrm{C}$, most of the vibrational features either disappear completely or significantly attenuate in intensity. ATR-IR spectra after calcination within 150-250 ${ }^{\circ} \mathrm{C}$ were almost identical, suggesting that following the drastic transformation at $T \geq 150{ }^{\circ} \mathrm{C}$, the vibrational structure was starkly modified, but this modified structure remained rather intact at temperatures above $150{ }^{\circ} \mathrm{C}$. The ATR-IR spectrum obtained after calcination at $250{ }^{\circ} \mathrm{C}$ (red spectrum in Figure $4 \mathrm{e})$ revealed two major modes that can survive upon calcination at temperatures above $150{ }^{\circ} \mathrm{C}$, a $\gamma_{\mathrm{C}-\mathrm{H}}$ (out-ofplane bending/opb) mode at $735 \mathrm{~cm}^{-1}$ and a $\delta_{\mathrm{C}-\mathrm{H}}$ (in-plane bending/ipb) mode at $1149 \mathrm{~cm}^{-1}$. These features indicate the presence of an aromatic organic coating on $\mathrm{Cu}$ NWs following the degradation of the BTA overlayer. Furthermore, bare $\mathrm{Cu}$ NWs (blue spectrum in Figure 4e) have IR features at 661 and $647 \mathrm{~cm}^{-1}$, which can be associated with copper oxide phonon modes $^{38-40}$ in line with the current XPS results (see XPS data for bare $\mathrm{Cu}$ NWs in Figure 4b). Therefore, the absence of such phonon modes for BTA-passivated Cu NW networks in Figure $4 \mathrm{~d}$,e suggests that BTA covers the $\mathrm{Cu}$ NW networks effectively without leaving a significant amount of uncovered/bare $\mathrm{Cu}$ NWs.

Chemical Corrosion Stability of Networks under the PEDOT:PSS Layer. Either as a hole injection or a hole transparent layer, transport electrodes are generally in contact with a PEDOT:PSS layer in organic-based optoelectronic devices. Most of the PEDOT:PSS types used in optoelectronic devices have relatively high acidity $(\mathrm{pH} \sim 3-4)$. Thus, the acidic nature of PEDOT:PSS eventually deteriorates the NW networks by acidic corrosion. ${ }^{41,42}$ As a result, long-term chemical corrosion stability against the acidic nature of PEDOT:PSS is another crucial requirement in industrial applications of transparent conducting electrodes. In this study, bare and BTA-passivated $\mathrm{Cu}$ NW networks were spincoated with a $50 \mathrm{~nm}$ thick PEDOT:PSS ( $\mathrm{pH} \sim 3$ ) layer. Then, the stability of both bare $\mathrm{Cu}$ NW/PEDOT:PSS and $\mathrm{Cu}$ NW/ BTA/PEDOT:PSS samples was monitored through sheet resistance measurements for 4 months. PEDOT:PSS is well known to be sensitive to air exposure, and its electrical conductivity degrades in time due to the absorption of atmospheric water/oxygen. Therefore, all samples were kept in a glovebox under a controlled argon atmosphere $\left(\mathrm{O}_{2}\right.$ level $<0.1$ ppm, $\mathrm{H}_{2} \mathrm{O}$ level $\left.<0.1 \mathrm{ppm}\right)$ throughout the test period to assure that the change in resistance was not related to the degradation of PEDOT:PSS. The initial average resistances for $\mathrm{Cu}$ NW/PEDOT:PSS samples and $\mathrm{Cu}$ NW/BTA/PEDOT:PSS samples were measured as $27 \pm 4$ and $25 \pm 2$, $\Omega /$ sq, respectively. At the end of 4 months, the resistance of $\mathrm{Cu}$ NW/PEDOT:PSS samples increased by approximately 500 times, while the resistance of $\mathrm{Cu}$ NW/BTA/PEDOT:PSS samples increased by only 5 times (Figure $5 \mathrm{a}$ ). It should also be noted that the maximum resistance of $\mathrm{Cu}$ NW/BTA/ PEDOT:PSS samples at the end of 4 months was below 150

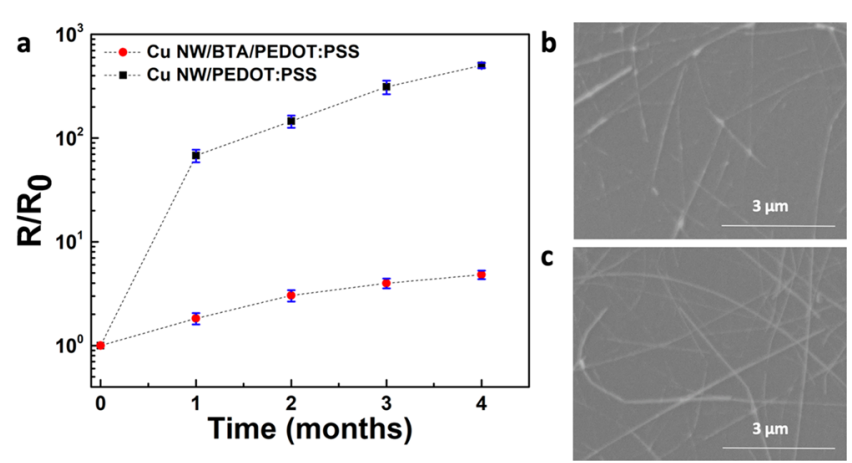

Figure 5. (a) Change in the sheet resistance of bare $\mathrm{Cu} \mathrm{NW} /$ PEDOT:PSS and Cu NW/BTA/PEDOT:PSS networks with time (lines are for visual aid). SEM images of (b) bare $\mathrm{Cu}$ NW/ PEDOT:PSS network and (c) Cu NW/BTA/PEDOT:PSS network after 4 months. 
a

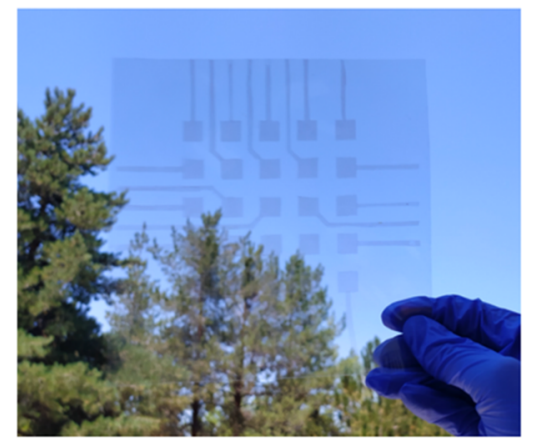

b

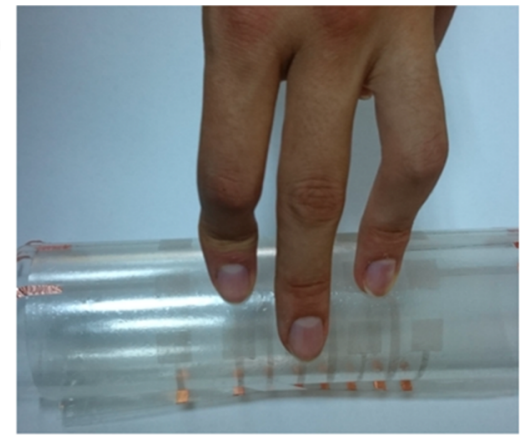

C

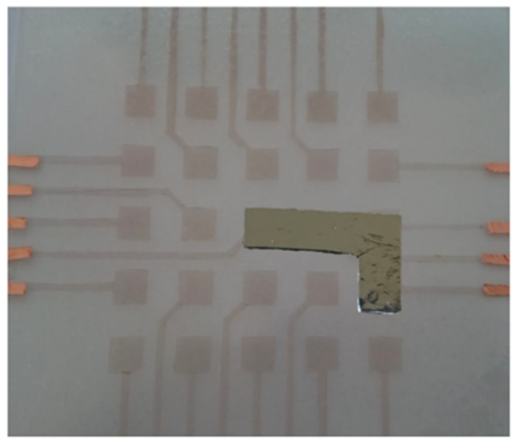

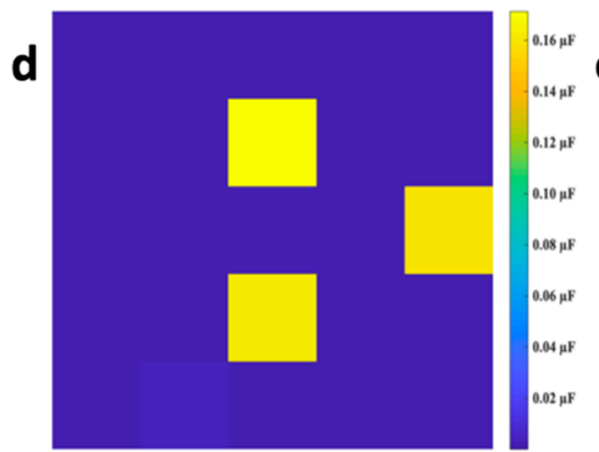
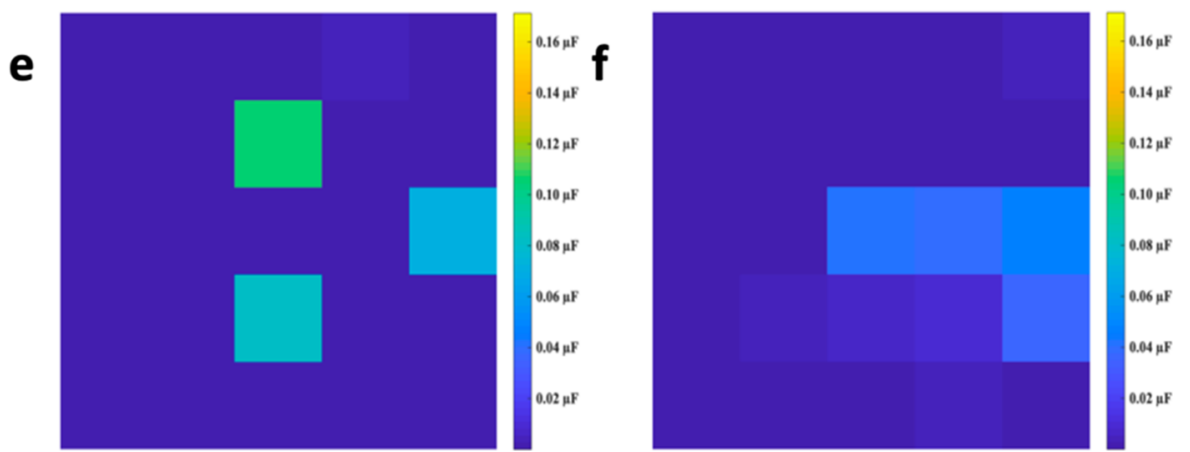

Figure 6. Photographs of the (a) fabricated transparent capacitive tactile sensor, (b) sensor upon three-finger touch when bent, and (c) sensor when an "L"-shaped conductive object is placed above. (d) Capacitance maps upon three-finger touch when the sensor was flat. (e) Capacitance map of (b). (f) Capacitance map of (c).

$\Omega /$ sq, which is still low enough for many optoelectronic applications. The obtained results were also supported by SEM analysis. The SEM image of the bare Cu NW/PEDOT:PSS sample showed that the structural integrity of the network was lost due to severe etching of the NWs (Figure 5b). Due to the acidic nature of PEDOT:PSS, bare $\mathrm{Cu}$ NWs were dissolved and ruptured within this acidic polymeric media. The overall electrical percolation within the network was destroyed, confirming the sheet resistance measurements. On the other hand, no morphological changes were observed for the $\mathrm{Cu}$ NW/BTA/PEDOT:PSS samples (Figure 5c). This implies that BTA-passivated samples show superior resistance against corrosion. The BTA layer deposited by a simple, solutionbased method suppresses the corrosion rate of $\mathrm{Cu}$ NWs under acidic conditions. These results are in perfect agreement with previous studies, where BTA treatment was shown to decrease the acidic corrosion rate of bulk $\mathrm{Cu} .{ }^{43}$

BTA-Passivated Cu NW-Based Capacitive Tactile Sensors. Transparent, flexible, capacitive tactile sensors were fabricated to demonstrate the potential of BTA-passivated $\mathrm{Cu}$ NW networks in optoelectronic device applications. Sensors were used to map the pressure applied by fingers and other objects with various shapes. Figures $6 \mathrm{a}-\mathrm{c}$ shows the photographs of the fabricated $5 \times 5$ matrix, single-electrode capacitive sensor devices.

For this capacitive sensor, in the loading mode, only one electrode was used to both transmit and receive signals. Electrodes were loaded capacitively by a conductive object and formed a displacement current flow through a conductive object to the ground. Bringing the conductive object closer to the sensor caused an increase in the capacitive coupling between the conductive object and the corresponding electrode. Changes in the magnitude of the output capacitance were proportional to the applied pressure. Figure S7 shows that the output capacitance of the fabricated sensors changes linearly with the applied pressure, where the sensitivity of the sensors was determined as $0.6 \times 10^{-3} \mu \mathrm{F} / \mathrm{kPa}$. An increase in the applied pressure decreased the thickness of the thermoplastic polyurethane (TPU) layer and increased the capacitance. The capacitance changes of 25 electrodes were recorded as a mapping figure. Figure $6 c, f$ showed an L-shaped conductive object (aluminum foil in this case) that was placed on the device and the corresponding response on the capacitance map, respectively. Contact (touch) information, position, and applied relative pressure of fingers or conductive objects interacting with the device could be obtained through the analysis of the increments in capacitance via mapping figures. As shown in Figure 6d, touching the tactile sensor with three fingers results in different capacitance values ranging from 0.15 to $0.17 \mu \mathrm{F}$.

The fabricated device was also capable of preserving its functionality under bending conditions. Figure $6 \mathrm{~b}$ shows the photo of the flexible sensor matrix attached to a transparent tube. Upon application of pressure to the sensor surface at three different points, similar and consistent map figures to those for the flat condition were obtained. Lower output capacitance values compared to that of the flat sensor were related to the incomplete coverage of the electrode area by fingers due to the curvature in the bent condition. It is worth noting that the control devices fabricated using bare $\mathrm{Cu}$ nanowire networks stopped working only 3 days after production. This signifies the necessity of the use of BTA for protecting these devices.

\section{CONCLUSIONS}

In summary, a simple, all-solution-based, and highly effective method to improve the resistance of $\mathrm{Cu} \mathrm{NW}$ networks against oxidation was demonstrated. In this method, bulk $\mathrm{Cu}$ 
corrosion inhibitor BTA was used for the passivation of $\mathrm{Cu}$ NW networks. It was demonstrated that BTA passivation does not affect the optoelectronic performance of networks adversely while providing outstanding oxidation resistance under various mild and harsh environments. Upon exposure to ambient conditions for 1 year, the sheet resistance of BTApassivated $\mathrm{Cu} \mathrm{NW}$ networks remained almost constant $\left(R / R_{0}<1.1\right)$. On the contrary, bare $\mathrm{Cu}$ NW networks became nonconductive after only 12 days. Furthermore, BTApassivated networks exhibited superior stability under longterm exposure to heat and humidity, showing a performance that drastically surpasses that of the bare control sample. In addition, BTA-passivated networks were successfully used as transparent and flexible electrodes for capacitive tactile sensors. Applications of the currently developed BTA-passivated $\mathrm{Cu}$ NW networks are not limited to tactile sensors, and they can be used in a wide range of different optoelectronic applications. The results obtained in the current study are of referring significance, as they provide a simple and scalable means to fabricate affordable $\mathrm{Cu}$ NW networks with high optoelectronic performance and excellent stability that can be utilized as promising alternatives to existing conventional materials used in optoelectronic devices.

\section{EXPERIMENTAL SECTION}

Chemicals. Hexadecylamine (HDA) $\left(\mathrm{C}_{16} \mathrm{H}_{35} \mathrm{~N}, \geq 94.0 \%(\mathrm{a} / \mathrm{a})\right)$, copper (II) chloride dihydrate $\left(\mathrm{CuCl}_{2} \cdot 2 \mathrm{H}_{2} \mathrm{O}, \geq 99.0 \%\right)$, D-(+)-glucose monohydrate (anhydrous, 97.5-102.0\%), poly(vinylpyrrolidone) (PVP) ( $\mathrm{MW}=55000$, monomer-based calculation), benzotriazole (BTA) $\left(\mathrm{C}_{6} \mathrm{H}_{5} \mathrm{~N}_{3} \geq 99.0 \%\right)$, chloroform $\left(\mathrm{CHCl}_{3}, 99.8 \%\right)$, and DL-lactic acid $(90 \%)$ were purchased from Sigma-Aldrich and used as received. Poly (3,4-ethylenedioxythiophene)-poly(styrenesulfonate) (PEDOT:PSS) with $\mathrm{pH} \sim 3$ was purchased from Heraeus (Heraeus VP Al 4083).

Synthesis of Cu NWs. Cu NWs were synthesized according to a previously reported procedure developed by Mohl et al. with slight modifications. ${ }^{44}$ In a typical synthesis, $147 \mathrm{mg}$ of $\mathrm{CuCl}_{2} \cdot 2 \mathrm{H}_{2} \mathrm{O}, 350$ $\mathrm{mg}$ of glucose, $1260 \mathrm{mg}$ of HDA, and $70 \mathrm{~mL}$ of deionized (DI) water (18.3 $\mathrm{M} \Omega$ ) were mixed in a glass beaker and stirred for $12 \mathrm{~h}$ via vigorous magnetic stirring at room temperature to obtain a homogeneous mixture. Afterward, the obtained light blue emulsion was transferred to a Teflon-lined autoclave with $100 \mathrm{~mL}$ capacity. The reactor was sealed and heated at $105{ }^{\circ} \mathrm{C}$ for $12 \mathrm{~h}$ without further stirring. Following synthesis, the NWs were purified by centrifugation and washed repeatedly with DI water and ethanol. Next, a multiphase separation method was carried out to eliminate the byproducts and to get rid of excess HDA. ${ }^{45}$ Following purification, the product was dispersed within a 2 wt \% PVP-ethanol solution. Excess PVP was removed by washing the NWs with ethanol. The final product was then dispersed in ethanol for further processing.

Fabrication and Passivation of Transparent Conducting Electrodes. Ethanolic suspensions of $\mathrm{Cu}$ NWs were deposited onto soda lime silica glass and poly(ethylene terephthalate) (PET) substrates using spray coating. $\mathrm{Cu}$ NW dispersion was sprayed onto the substrates at $100{ }^{\circ} \mathrm{C}$ using an airbrush fed by pure nitrogen gas at $1.5 \mathrm{~atm}$. Following deposition, $\mathrm{Cu} \mathrm{NW}$ networks on glass were annealed under vacuum at $200{ }^{\circ} \mathrm{C}$ for $20 \mathrm{~min}$ to get rid of the residual PVP layer and to improve the electrical contact at junctions of $\mathrm{Cu}$ NWs. Electrodes on PET were immersed into a lactic acid-ethanol solution at a volume ratio of $1: 20$, which was followed by an ethanol rinse and drying. In the meantime, a 3 wt \% BTA-ethanol solution was prepared by stirring at $300 \mathrm{rpm}$ for $10 \mathrm{~min}$ in a tightly sealed glass vial at room temperature. The solution was then spin-coated onto $\mathrm{Cu}$ NW networks at a speed of $3000 \mathrm{rpm}$ for $30 \mathrm{~s}$ after which the samples were dried at $70{ }^{\circ} \mathrm{C}$ for $30 \mathrm{~min}$. After $24 \mathrm{~h}$, the samples were rinsed with ethanol to get rid of any excess BTA.
Fabrication of the Capacitive Tactile Sensor. For the fabrication of the sensor, BTA-passivated $\mathrm{Cu}$ NW networks on PET substrates were used and a $240 \mu \mathrm{m}$ thick TPU was attached to it as a dielectric layer and mechanical support. Each electrode with a size of $1 \times 1 \mathrm{~cm}^{2}$ was connected with a load resistor and then a microcontroller unit (MCU). The schematic circuit of the fabricated capacitive sensor for one electrode is shown in Figure S8. The timer inside the MCU calculates the capacitance of the system through elapsed time while pressing.

Characterizations and Measurements. SEM was performed with an FEI NOVA NANO SEM 430 operated at $10 \mathrm{kV}$. Transmission electron microscopy (TEM) images were obtained on a JEOL TEM $2100 \mathrm{~F}$ operated at $200 \mathrm{kV}$. X-ray photoelectron spectroscopy (XPS) measurements were performed using SPECS PHOIBOS and PHI 5000 Versa Probe X-ray photoelectron spectrometers with monochromatic $\mathrm{Al} \mathrm{K} \alpha \mathrm{X}$-ray excitation sources $(15 \mathrm{kV}, 400 \mathrm{~W}, 1486.6 \mathrm{eV})$ and hemispherical photoelectron energy analyzers. Binding energy (B.E.) calibration was made by setting the adventitious carbon (C 1s) signal to $284.4 \mathrm{eV}$. Peak fitting, baseline correction, and deconvolution analysis of the XPS data were performed via CASA and OriginPro software. ATR-IR measurements were performed on a Bruker Alpha spectrometer equipped with a Bruker ATR attachment and a DLaTGS detector. Each ATR-IR spectrum was acquired with a frequency resolution of $4 \mathrm{~cm}^{-1}$ by averaging 32 scans. To eliminate atmospheric water and carbon dioxide vibrational signatures, a background spectrum of air was acquired before each sample spectrum.

Sheet resistance and transparency measurements were performed using a Keithley 2400 source meter and an Agilent 8453 UV-vis spectrophotometer, respectively. Continuous humidity and temperature measurements were performed with a chamber-mounted remote Weather Forecast digital hygrometer probe (temperature range $=\sim 0$ to $+50{ }^{\circ} \mathrm{C}$, thermometer accuracy $= \pm 1{ }^{\circ} \mathrm{C}$, humidity range $=20-$ $98 \% \mathrm{RH}$, humidity accuracy $= \pm 5 \%$ ).

\section{ASSOCIATED CONTENT}

\section{Supporting Information}

The Supporting Information is available free of charge at https://pubs.acs.org/doi/10.1021/acsami.0c11729.

Scanning Electron Microscope (SEM) images, statistical information (diameter and length distribution histograms of nanowires), additional X-ray photoelectron spectroscopy (XPS) results, areal mass density (amd) calculation procedure, and graph related to the tactile sensor capacitance change with respect to pressure (PDF)

\section{AUTHOR INFORMATION}

\section{Corresponding Author}

Husnu Emrah Unalan - Department of Metallurgical and Materials Engineering, Department of Micro and Nanotechnology, and Centre for Solar Energy Research and Applications (GÜNAM), Middle East Technical University, Ankara 06800, Turkey; 이이. orcid.org/0000-0003-3667-179X; Email: unalan@metu.edu.tr

\section{Authors}

Sevim Polat Genlik - Department of Materials Science and Engineering, Ohio State University, Columbus, Ohio 43210, United States

Dogancan Tigan - Department of Metallurgical and Materials Engineering, Middle East Technical University, Ankara 06800, Turkey

Yusuf Kocak - Department of Chemistry, Bilkent University,

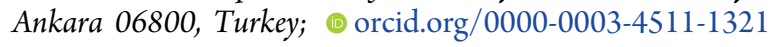


Kerem Emre Ercan - Department of Chemistry, Bilkent University, Ankara 06800, Turkey; (i) orcid.org/0000-00034650-7977

Melih Ogeday Cicek - Department of Metallurgical and Materials Engineering, Middle East Technical University, Ankara 06800, Turkey

Sensu Tunca - Department of Metallurgical and Materials Engineering, Middle East Technical University, Ankara 06800, Turkey

Serkan Koylan - Department of Metallurgical and Materials Engineering, Middle East Technical University, Ankara 06800, Turkey

Sahin Coskun - Department of Metallurgical and Materials Engineering, Eskisehir Osmangazi University, Eskisehir 26040, Turkey

Emrah Ozensoy - Department of Chemistry and UNAMNational Nanotechnology Center, Bilkent University, Ankara 06800, Turkey; ○ orcid.org/0000-0003-4352-3824

Complete contact information is available at: https://pubs.acs.org/10.1021/acsami.0c11729

\section{Author Contributions}

H.E.U. and E.O. supervised the research. S.P.G. and D.T. planned and performed the experiments. S.P.G. collected and analyzed the data and wrote the paper. Y.K. and K.E.E. helped with characterization and discussed the data. M.O.C., S.T., and S.K. performed the experiments to show the functionality of the discussed material. S.C. provided scientific discussions to give direction to the research. All authors have discussed, commented on, and revised the manuscript.

\section{Notes}

The authors declare no competing financial interest.

\section{ACKNOWLEDGMENTS}

This work was financially supported by the Middle East Technical University through the Scientific Research Projects Programme under project numbers BAP-07-02-2016-003, BAP-07-02-2017-011, and BAP-07-02-2017-004-265. E.O. acknowledges the scientific collaboration with the TARLA project founded by the Ministry of Development of Turkey (project code DPT2006K-120470). The authors also acknowledge Mr. Mete Duman (UNAM-National Nanotechnology Center, Ankara, Turkey) for the design and production of technical schematics.

\section{REFERENCES}

(1) Bellet, D.; Lagrange, M.; Sannicolo, T.; Aghazadehchors, S.; Nguyen, V. H.; Langley, D. P.; Munoz-Rojas, D.; Jimenez, C.; Brechet, Y.; Nguyen, N. D. Transparent Electrodes Based on Silver Nanowire Networks: From Physical Considerations Towards Device Integration. Materials 2017, 10, 570.

(2) Hosono, Hideo.; David, C. P. Handbook of Transparent Conductors; Springer Science \& Business Media, 2010.

(3) Coskun, S.; Ates, E. S.; Unalan, H. E. Optimization of Silver Nanowire Networks for Polymer Light Emitting Diode Electrodes. Nanotechnology 2013, 24, No. 125202.

(4) Ye, S.; Rathmell, A. R.; Chen, Z.; Stewart, I. E.; Wiley, B. J. Metal Nanowire Networks: The Next Generation of Transparent Conductors. Adv. Mater. 2014, 26, 6670-6687.

(5) Sannicolo, T.; Lagrange, M.; Cabos, A.; Celle, C.; Simonato, J. P.; Bellet, D. Metallic Nanowire-Based Transparent Electrodes for Next Generation Flexible Devices: a Review. Small 2016, 12, 60526075 .
(6) Kumar, A.; Zhou, C. The Race To Replace Tin-Doped Indium. ACS Nano 2010, 4, 11-14.

(7) Niu, Z.; Cui, F.; Yu, Y.; Becknell, N.; Sun, Y.; Khanarian, G.; Kim, D.; Dou, L.; Dehestani, A.; Schierle-Arndt, K.; Yang, P. Ultrathin Epitaxial Cu@Au Core-Shell Nanowires for Stable Transparent Conductors. J. Am. Chem. Soc. 2017, 139, 7348-7354.

(8) Stewart, I. E.; Rathmell, A. R.; Yan, L.; Ye, S.; Flowers, P. F.; You, W.; Wiley, B. J. Solution-Processed Copper-Nickel Nanowire Anodes for Organic Solar Cells. Nanoscale 2014, 6, 5980-5988.

(9) Won, Y.; Kim, A.; Lee, D.; Yang, W.; Woo, K.; Jeong, S.; Moon, J. Annealing-Free Fabrication of Highly Oxidation-Resistive Copper Nanowire Composite Conductors for Photovoltaics. NPG Asia Mater. 2016, 6, No. e105.

(10) Chen, J.; Zhou, W.; Chen, J.; Fan, Y.; Zhang, Z.; Huang, Z.; Feng, X.; Mi, B.; Ma, Y.; Huang, W. Solution-Processed Copper Nanowire Flexible Transparent Electrodes with PEDOT:PSS as Binder, Protector and Oxide-Layer Scavenger for Polymer Solar Cells. Nano Res. 2015, 8, 1017-1025.

(11) Im, H. G.; Jung, S. H.; Jin, J.; Lee, J.; Lee, D.; Lee, J. Y.; Kim, I. D.; Bae, B. S.; Lee, D. Flexible Transparent Conducting Hybrid Film Using a Surface-Embedded Copper Nanowire Network: A Highly Oxidation-Resistant Copper Nanowire Electrode for Flexible Optoelectronics. ACS Nano 2014, 8, 10973-10979.

(12) Song, J.; Li, J.; Xu, J.; Zeng, H. Superstable Transparent Conductive $\mathrm{Cu} @ \mathrm{Cu}_{4} \mathrm{Ni}$ Nanowire Elastomer Composites Against Oxidation, Bending, Stretching, and Twisting for Flexible and Stretchable Optoelectronics. Nano Lett. 2014, 14, 6298-6305.

(13) Wang, J.; Chen, H.; Zhao, Y.; Zhong, Z.; Tang, Y.; Liu, G.; Kang, J.; et al. Programmed Ultrafast Scan Welding of Cu Nanowires Network with Pulsed Ultraviolet Laser Beam for Transparent Conductive Electrodes and Flexible Circuits. ACS Appl. Mater. Interfaces 2020, 12, 35211-35221.

(14) Chu, H. C.; Chang, Y. C.; Lin, Y.; Chang, S. H.; Chang, W. C.; Li, G. A.; Tuan, H. Y. Spray-Deposited Large-Area Copper Nanowire Transparent Conductive Electrodes and Their Uses for Touch Screen Applications. ACS Appl. Mater. Interfaces 2016, 8, 13009-13017.

(15) Bao, C.; Yang, J.; Gao, H.; Li, F.; Yao, Y.; Yang, B.; Fu, G.; Zhou, X.; Yu, T.; Qin, Y.; Liu, J.; Zou, Z. In Situ Fabrication of Highly Conductive Metal Nanowire Networks with High Transmittance From Deep-Ultraviolet to Near-Infrared. ACS Nano 2015, 9, 25022509.

(16) Celle, C.; Cabos, A.; Fontecave, T.; Laguitton, B.; Benayard, A.; Guettaz, L.; Pelissier, N.; Nguyen, V. H.; Bellet, D.; Munoz-Rojas, D.; Simonato, J. P. Oxidation of Copper Nanowire Based Transparent Electrodes in Ambient Conditions and Their Stabilization by Encapsulation: Application to Transparent Film Heaters. Nanotechnology 2018, 29, No. 085701.

(17) Zhai, H.; Wang, R.; Wang, X.; Cheng, Y.; Shi, L.; Sun, J. Transparent Heaters Based on Highly Stable Cu Nanowire Films. Nano Res. 2016, 9, 3924-3936.

(18) Stewart, I. E.; Ye, S.; Chen, Z.; Flowers, P. F.; Wiley, B. J. Synthesis of $\mathrm{Cu}-\mathrm{Ag}, \mathrm{Cu}-\mathrm{Au}$, and $\mathrm{Cu}-\mathrm{Pt}$ Core-Shell Nanowires and Their Use in Transparent Conducting Films. Chem. Mater. 2015, 27, $7788-7794$.

(19) Rathmell, A. R.; Nguyen, M.; Chi, M.; Wiley, B. J. Synthesis of Oxidation-Resistant Cupronickel Nanowires for Transparent Conducting Nanowire Networks. Nano Lett. 2012, 12, 3193-3199.

(20) Chen, J.; Chen, J.; Li, Y.; Zhou, W.; Feng, X.; Huang, Q.; Zheng, J.; Liu, R.; Ma, Y.; Huang, W. Enhanced Oxidation-resistant $\mathrm{Cu}-\mathrm{Ni}$ Core-Shell Nanowires: Controllable One-Pot Synthesis and Solution Processing to Transparent Flexible Heaters. Nanoscale 2015, 7, 16874-16879.

(21) Hsu, P. C.; Wu, H.; Carney, T. J.; McDowell, M. T.; Yang, Y.; Garnett, E. C.; Li, M.; Hu, L.; Cui, Y. Passivation Coating on Electrospun Copper Nanofibers for Stable Transparent Electrodes. ACS Nano 2012, 6, 5150-5156.

(22) Chen, Z.; Ye, S.; Stewart, I. E.; Wiley, B. J. Copper Nanowire Networks with Transparent Oxide Shells That Prevent Oxidation without Reducing Transmittance. ACS Nano 2014, 8, 9673-9679. 
(23) Shi, L.; Wang, R.; Zhai, H.; Liu, Y.; Gao, L.; Sun, J. A LongTerm Oxidation Barrier for Copper Nanowires: Graphene Says Yes. Phys. Chem. Chem. Phys. 2015, 17, 4231-4236.

(24) Dou, L.; Cui, F.; Yu, Y.; Khanarian, G.; Eaton, S. W.; Yang, Q.; Resasco, J.; Schildknecht, C.; Schierle-Arndt, K.; Yang, P. SolutionProcessed Copper/Reduced- Graphene-Oxide Core/Shell Nanowire Transparent Conductors. ACS Nano 2016, 10, 2600-2606.

(25) Liu, G.; Wang, J.; Ge, Y.; Wang, Y.; Lu, S.; Zhao, Y.; Tang, Y.; Soomro, A. M.; Hong, Q.; Yang, X.; Xu, F.; Li, S.; Chen, L. J.; Cai, D.; Kang, J. Cu Nanowires Passivated with Hexagonal Boron Nitride-An Ultra-Stable, Selectively Transparent Conductor. ACS Nano 2020, 14, 6761-6773.

(26) Ahn, Y.; Lee, D.; Jeong, Y.; Lee, H.; Lee, Y. Flexible Metal Nanowire-Parylene C Transparent Electrodes for Next Generation Optoelectronic Devices. J. Mater. Chem. C 2017, 5, 2425-2431.

(27) Fateh, A.; Aliofkhazraei, M.; Rezvanian, A. R. Review of Corrosive Environments for Copper and Its Corrosion Inhibitors. Arabian J. Chem. 2020, 13, 481-544.

(28) Brusic, V.; et al. Copper Corrosion With and Without Inhibitors. J. Electrochem. Soc. 1991, 138, No. 2253.

(29) Cotton, J. B.; Scholes, I. R. Benzotriazole and Related' Compounds As. Brit. Corros. J. 1967, 2, 1-5.

(30) Xue, G.; Ding, J. Chemisorption of a Compact Polymeric Coating on Copper Surfaces From a Benzotriazole Solution. Appl. Surf. Sci. 1990, 40, 327-332.

(31) Chadwick, D.; Hashemi, T. Adsorbed Corrosion Inhibitors Studied by Electron Spectroscopy: Benzotriazole on Copper and Copper Alloys. Corros. Sci. 1978, 18, 39-51.

(32) Jiang, P.; Prendergast, D.; Borondics, F.; Porsgaard, S.; Giovanetti, L.; Pach, E.; Salmeron, M.; et al. Experimental and Theoretical Investigation of the Electronic Structure of $\mathrm{Cu}_{2} \mathrm{O}$ and $\mathrm{CuO}$ Thin Films on $\mathrm{Cu}$ (110) Using X-ray Photoelectron and Absorption Spectroscopy. J. Chem. Phys. 2013, 138, No. 024704.

(33) Susman, M. D.; Feldman, Y.; Vaskevich, A.; Rubinstein, I. Chemical Deposition and Stabilization of Plasmonic Copper Nanoparticle Films on Transparent Substrates. Chem. Mater. 2012, 24, 2501-2508.

(34) Stewart, I. E. The Impact of Morphology and Composition on the Resistivity and Oxidation Resistance of Metal Nanostructure Films. PhD Thesis, Duke University, 2016.

(35) Popova, I.; Yates, J. T. Adsorption and Thermal Behavior of Benzotriazole Chemisorbed on $\gamma-\mathrm{Al}_{2} \mathrm{O}_{3}$. Langmuir 1997, 13, 61696175.

(36) Hajimammadov, R.; Mohl, M.; Kordas, K. Native Oxide Formation on Pentagonal Copper Nanowires: A TEM Study. Surf. Sci. 2018, 672-673, 19-22.

(37) Christensen, T. M.; Sorensen, N. R. Thermal Stability of Benzotriazole on Copper During Atmospheric Corrosion. Surf. Interface Anal. 1991, 17, 3-6.

(38) Liu, D.; Yang, S.; Lee, S. T. Preparation of Novel Cuprous Oxide- Fullerene [60] Core-Shell Nanowires and Nanoparticles via a Copper (I)-Assisted Fullerene-Polymerization Reaction. The. J. Phys. Chem. C 2008, 112, 7110-7118.

(39) Tammer, M.; Sokrates, G. Infrared and Raman Characteristic Group Frequencies: Tables and Charts; John Wiley \& Sons, 2004.

(40) Akgul, F. A.; Akgul, G.; Yildirim, N.; Unalan, H. E.; Turan, R. Influence of Thermal Annealing on Microstructural, Morphological, Optical Properties and Surface Electronic Structure of Copper Oxide Thin Films. Mater. Chem. Phys. 2014, 147, 987-995.

(41) Song, T. B.; Rim, Y. S.; Liu, F.; Bob, B.; Le, S.; Hsieh, Y. T.; Yang, Y. Highly Robust Silver Nanowire Network for Transparent Electrode. ACS Appl. Mater. Interfaces 2015, 7, 24601-24607.

(42) Choi, D. Y.; Kang, H. W.; Sung, H. J.; Kim, S. S. AnnealingFree, Flexible Silver Nanowire-Polymer Composite Electrodes via a Continuous Two-Step Spray-Coating Method. Nanoscale 2013, 5, 977-983.

(43) Ullah, S.; Shariff, A. M.; Bustam, M. A.; Nadeem, M.; Naz, M. Y.; Ayoub, M. Study on Effect of Benzotriazole and Surfactants on
Corrosion Inhibition of Copper Alloys in Sulphuric acid. Int. J. Electrochem. Sci. 2015, 10, 9443-9455.

(44) Mohl, M.; Pusztai, P.; Kukovecz, A.; Konya, Z.; Kukkola, J.; Kordas, K.; Vajtai, R; Ajayan, P. M. Low-Temperature Large-Scale Synthesis and Electrical Testing of Ultralong Copper Nanowires. Langmuir 2010, 26, 16496-16502.

(45) Qian, F.; Lan, P. C.; Olson, T.; Zhu, C.; Duoss, E. B.; Spadaccini, C. M.; Han, T. Y. J. Multiphase Separation of Copper Nanowires. Chem. Commun. 2016, 52, 11627-11630. 